\title{
Sliding dichotomy compared with fixed dichotomization of ordinal outcome scales in subarachnoid hemorrhage trials
}

\author{
Clinical article
}

\author{
Don Ilodigwe, M.Stat., ${ }^{1,2}$ Gordon D. Murray, Ph.D.,${ }^{3}$ Neal F. Kassell, M.D. ${ }^{4}$ \\ James Torner, Ph.D., ${ }^{5}$ Richard S. C. Kerr, M.D., ${ }^{6}$ Andrew J. MolyneuX, F.R.C.R., ${ }^{7}$ \\ and R. Loch Macdonald, M.D., Ph.D.,
}

${ }^{1}$ Division of Neurosurgery, St. Michael's Hospital, Labatt Family Centre of Excellence in Brain Injury and Trauma Research, Keenan Research Centre in the Li Ka Shing Knowledge Institute of St. Michael's Hospital; ${ }^{2}$ Department of Surgery, Institute of Medical Science, University of Toronto, Ontario, Canada; ${ }^{4}$ Department of Neurological Surgery, University of Virginia School of Medicine, Charlottesville, Virginia; ${ }^{5}$ Department of Epidemiology, University of Iowa, College of Public Health, Iowa City, Iowa; ${ }^{3}$ Centre for Population Health Sciences, University of Edinburgh Medical School, Edinburgh; ${ }^{6}$ Department of Neurosurgery, West Wing, John Radcliffe Hospital, Oxford; and ${ }^{7}$ Oxford Neurovascular and Neuroradiology Research Unit, University of Oxford, United Kingdom

Object. In randomized clinical trials of subarachnoid hemorrhage (SAH) in which the primary clinical outcomes are ordinal, it has been common practice to dichotomize the ordinal outcome scale into favorable versus unfavorable outcome. Using this strategy may increase sample sizes by reducing statistical power. Authors of the present study used SAH clinical trial data to determine if a sliding dichotomy would improve statistical power.

Methods. Available individual patient data from tirilazad (3552 patients), clazosentan (the Clazosentan to Overcome Neurological Ischemia and Infarction Occurring After Subarachnoid Hemorrhage trial [CONSCIOUS-1], 413 patients), and subarachnoid aneurysm trials (the International Subarachnoid Aneurysm Trial [ISAT], 2089 patients) were analyzed. Treatment effect sizes were examined using conventional fixed dichotomy, sliding dichotomy (logical or median split methods), or proportional odds modeling. Whether sliding dichotomy affected the difference in outcomes between the several age and neurological grade groups was also evaluated.

Results. In the tirilazad data, there was no significant effect of treatment on outcome (fixed dichotomy: OR $=0.92,95 \%$ CI 0.80-1.07; and sliding dichotomy: OR $=1.02,95 \%$ CI 0.87-1.19). Sliding dichotomy reversed and increased the difference in outcome in favor of the placebo over clazosentan (fixed dichotomy: OR $=1.06,95 \%$ CI $0.65-1.74$; and sliding dichotomy: OR $=0.85,95 \%$ CI $0.52-1.39$ ). In the ISAT data, sliding dichotomy produced identical odds ratios compared with fixed dichotomy (fixed dichotomy vs sliding dichotomy, respectively: OR $=0.67,95 \%$ CI $0.55-0.82$ vs $\mathrm{OR}=0.67,95 \% \mathrm{CI}$ $0.53-0.85)$. When considering the tirilazad and CONSCIOUS-1 groups based on age or World Federation of Neurosurgical Societies grade, no consistent effects of sliding dichotomy compared with fixed dichotomy were observed.

Conclusions. There were differences among fixed dichotomy, sliding dichotomy, and proportional odds models in the magnitude and precision of odds ratios, but these differences were not as substantial as those seen when these methods were used in other conditions such as head injury. This finding suggests the need for different outcome scales for SAH.

(http://thejns.org/doi/abs/10.3171/2012.9.JNS111383)

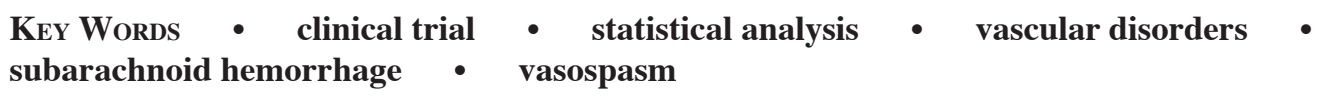

$\mathrm{O}$ RDINAL outcome scales, such as the $\mathrm{GOS}^{7}$ and $\mathrm{mRS},{ }^{3,15}$ have been used in phase III randomized clinical trials to measure clinical outcome. The standard statistical analysis collapses outcomes into 2

Abbreviations used in this paper: CONSCIOUS-1 = Clazosentan to Overcome Neurological Ischemia and Infarction Occurring After Subarachnoid Hemorrhage; GOS = Glasgow Outcome Scale; GOS-E = extended GOS; ISAT = International Subarachnoid Aneurysm Trial; $\mathrm{mRS}=$ modified Rankin Scale; $\mathrm{SAH}=$ subarachnoid hemorrhage; WFNS = World Federation of Neurosurgical Societies. categories defined as favorable and unfavorable. For example, the GOS is usually subdivided as favorable (moderate disability and good recovery) and unfavorable (severe disability, vegetative state, and death). The $\mathrm{mRS}$ is dichotomized at various points such as $0-1$ versus $2-6$ or 0-2 versus 3-6. ${ }^{4}$ This approach makes statistical analysis and interpretation of the results simple but does have theoretical disadvantages of loss of information about outcome and reduced statistical power.

Other methods have been proposed to avoid these issues. One is to use the same end point but to statistically analyze it by some method that treats the categories as 
ordinal, such as ordinal logistic regression. ${ }^{1}$ Ordinal logistic regression analyzes the dependent variable, which in our case is clinical outcome, as 3 or more categories and takes into account the inherent ordering of the groups (that is, outcome is progressively worse as one goes down the scale). Proportional odds modeling is a type of ordinal logistic regression that compares the dependent outcome variable at each of the possible dichotomous breakpoints in the ordinal scale. The proportional odds model assumes that, for example, in the case of the 5-point GOS, the odds ratio of a favorable versus unfavorable outcome is the same for each of the 4 possible dichotomous breakpoints in the GOS. Ordinal logistic regression models have been called "shift analysis." 20

Other methods include the global statistic and responder analysis. ${ }^{17,20}$ These methods have not been used in clinical trials of SAH. Responder analysis using the sliding dichotomy strategy has been retrospectively applied to head injury data and used as the primary outcome analysis in a trial of intracerebral hemorrhage. ${ }^{14,17}$ With this method, the definition of favorable outcome depends on the baseline condition of the patient. Thus, a young patient with a WFNS grade of I might be classified as having a favorable outcome only if the patient made a good recovery, whereas the mere survival of a patient with WFNS Grade V might be classified as a favorable outcome. Sliding dichotomy is implemented by estimating prognosis for a patient at baseline and dividing it into 3 or more sequential tiers based on predicted outcome. Each tier is then dichotomized into favorable and unfavorable outcome categories either at some logical cut point (sliding approach) or nearest the point that divides the tier into an equal number of patients with favorable and unfavorable outcomes (median approach).

We hypothesized that some insight into the potential utility of these methods could be obtained by reanalyzing existing SAH clinical trial databases using these alternative methods and comparing the results to standard dichotomous statistics.

\section{Methods}

\section{Patient Population}

Available individual patient data from 7 trials were obtained from 6054 patients with aneurysmal SAH, including 4 trials of tirilazad, ${ }^{5,6,8-10} 1$ trial of clazosentan (CONSCIOUS-1), ${ }^{12}$ and 1 trial comparing clipping and coiling (ISAT; Table 1). ${ }^{15}$

The tirilazad studies (3552 patients) were conducted in neurosurgical centers in Europe, Australia, New Zealand, South Africa, and North America between 1990 and 1997. These were randomized, double-blind placebocontrolled trials using various doses of tirilazad. All patients had angiographically documented aneurysms causing SAH confirmed by CT or lumbar puncture and were at least 18 years old and able to begin treatment within 48 hours of SAH. The main difference in entry criteria among the 4 tirilazad studies was that only females were entered in the last 2 studies. The outcome measure was clinical outcome at 3 months posttreatment as measured on the GOS. The 54 patients with missing 3-month GOS data were excluded from analysis. Almost all patients were treated via neurosurgical clipping.

The second set of patient data were from a post hoc exploratory analysis of CONSCIOUS-1 (http://clinicaltrials. gov/ct/show/NCT00111085). ${ }^{12}$ The methods and results of this randomized, placebo-controlled double-blind study have been described elsewhere. Outcome was assessed via a centrally administered telephone interview 12 weeks after SAH and was measured using the extended version of the GOS (GOS-E). ${ }^{22}$ Forty-five percent of the patients underwent neurosurgical clipping of the ruptured aneurysm, with the remainder treated via endovascular coiling.

The final set of patient data was from the ISAT, which included 2089 patients with aneurysmal SAH who were randomized to undergo neurosurgical clipping or endovascular coiling of the ruptured aneurysm. ${ }^{55,16}$ The outcome measure was the mRS, and scores were assessed by telephone interview or in person 12 months after SAH.

\section{Statistical Analysis}

We first used binary logistic regression to estimate the effects of tirilazad and clazosentan on the dichotomous GOS or GOS-E measured at 3 months posttreatment, respectively. The GOS and GOS-E were dichotomized into favorable (good recovery or moderate disability) or unfavorable (severe disability, vegetative state, or death). For the ISAT, the effect of the method of treatment (clipping vs coiling) on the dichotomous mRS was first estimated using binary logistic regression. The mRS was dichotomized into favorable (mRS 0-2 [good recovery, no significant disability, slight disability]) or unfavorable (mRS 3-6 [moderate disability, moderately severe disability, severe disability, or death]). These analyses were adjusted for baseline covariates, which were age, SAH clot thickness, preexisting hypertension, and WFNS grade for the tirilazad data; age, sex, SAH clot thickness, and preexisting hypertension for the CONSCIOUS-1 data; and age, sex, and WFNS grade for the ISAT data. The rationale for these adjustments was that multivariate logistic regression using the dichotomous GOS, GOS-E, or mRS identified these factors in the respective data sets as significant independent variables associated with outcome $(\mathrm{p}<0.05)$.

Next, we used 3 other approaches to analyze the outcomes: proportional odds logistic regression modeling and a sliding dichotomy (implemented using logical cut points or median split methods). The proportional odds logistic regression models were fitted with the GOS or GOS-E considered as a 5-, 4-, or 3-point ordinal scale (vegetative state and death were always combined). The mRS was considered as a 7-, 6-, 5-, 4-, or 3-point ordinal scale. Proportional odds logistic regression estimates a common odds ratio for each of the possible cut points of the outcome scale. The common odds ratio is formally valid if the odds ratios for each cut point are the same (that is, the proportional odds assumption). The common odds ratio can be interpreted as a summary measure of treatment effect, even though the odds ratios may differ between the different cut points. ${ }^{21}$

The sliding dichotomy also dichotomizes outcome 
TABLE 1: Characteristics of patients from the tirilazad and CONSCIOUS-1 trials and the ISAT*

\begin{tabular}{|c|c|c|c|c|c|c|}
\hline \multirow[b]{2}{*}{ Variable } & \multicolumn{2}{|c|}{ Tirilazad Trials } & \multicolumn{2}{|c|}{ CONSCIOUS-1 Trial } & \multicolumn{2}{|c|}{ ISAT } \\
\hline & Tirilazad & Placebo & Clazosentan & Placebo & Clipping & Coiling \\
\hline no. of patients & 2174 & 1378 & 317 & 96 & 1041 & 1048 \\
\hline mean age in yrs & $52 \pm 13$ & $52 \pm 13$ & $51 \pm 11$ & $52 \pm 11$ & $52 \pm 12$ & $52 \pm 12$ \\
\hline WFNS Grade I-II & $1694(78)$ & $1057(77)$ & $246(78)$ & $67(70)$ & $996(96)$ & $999(95)$ \\
\hline no. of females & $1715(79)$ & $1218(88)$ & $228(72)$ & $64(67)$ & $655(63)$ & $660(63)$ \\
\hline thick SAH & $1398(64)$ & $917(67)$ & $238(75)$ & $70(73)$ & $807(78)$ & $803(77)$ \\
\hline
\end{tabular}

* Values represent the means \pm standard deviations or the number of patients (\%).

(GOS, GOS-E, or mRS in this case) into a binary measure, but the cut point defining favorable or unfavorable outcome depends on the predicted prognosis for an individual patient on entry into the trial. ${ }^{17,21}$ For example, for a patient who is predicted to have a good recovery, only a good recovery might be considered a favorable outcome, whereas in the traditional dichotomy such a patient could be moderately disabled but still classified as having a favorable outcome. Similarly, for a patient with a very poor prognosis who is predicted to die or be in a vegetative state, severe disability might be regarded as a favorable outcome. For sliding dichotomy, we first estimated the baseline prognostic risk in each patient by calculating the probability of a favorable outcome using a predictive model with the above-listed baseline variables adjusted for in each data set in the initial logistic regression modeling. Then, based on the tertiles of prognostic scores, patients were grouped into 3 prognostic bands of approximately equal size, representing the best, intermediate, and worst prognosis. For each band, a separate cut point on either the GOS/GOS-E or the mRS was defined and a new outcome variable was created. The cut points were determined using either a sliding or logical cut point, or a median split approach. For a sliding or logical cut point, favorable outcome was defined in the worst tier as severe or moderate disability or good recovery on the 5-point GOS, moderate disability or good recovery in the intermediate tier, and good recovery in the best tier. For the mRS, favorable outcome in the worst tier included scores of $0-4$, scores of $0-3$ in the intermediate tier, and scores of $0-2$ in the best tier. For the median approach, the point for dichotomization of favorable versus unfavorable outcome in each tier was defined as close as possible to the breakpoint between categories of outcome in the tier that was closest to producing a 50:50 split in favorable and unfavorable outcomes in that tier. These breakpoints were different for each data set. Furthermore, the breakpoints were the same for some tiers because of the skewed distribution of outcomes. The effect of treatment on this newly generated dichotomous outcome was then estimated using binary logistic regression, with stratification by prognostic bands and adjustment for the baseline covariates mentioned above. The pooled sliding dichotomy odds ratio could then be interpreted as the effect of treatment on outcome.

Since treatment with tirilazad or clazosentan did not significantly affect outcome on the GOS or GOS-E, respectively, each of the above analyses was repeated using age ( $\leq 51$ or $>51$ years old) and WFNS grade (I-III or IV-V) as the treatment variable, based on the premise that these groups have different outcomes and thus that proportional odds models or sliding dichotomy might change the relative differences in outcome between these groups.

We calculated Wald statistics based on the coefficients of the treatment effect and the corresponding standard error for each analysis. The ratio of the Wald statistics can be interpreted as a gain in information density and is therefore a suitable measure for the efficiency of the different approaches in analyzing outcomes.

Finally, we performed Monte Carlo simulations on the patient-level data extracted from the tirilazad and ISAT data sets, generating a simulated sample of 1000 patients. For a single simulation with any one of our trial databases (tirilazad or ISAT), 500 patients were randomly sampled with replacement from the control group (placebo in the tirilazad trials, clipping in the ISAT) and another 500 patients from the treatment group (tirilazad in the tirilazad trials, coiling in the ISAT) to generate simulated sample data of 1000 patients. Simulated data were then reanalyzed with each of the different approaches, and their predictive powers were compared. For analyses focused on either the conventional or sliding dichotomy approach, we estimated the parameters (OR with 95\% confidence interval, Wald statistic, and $p$ value) separately for each simulation by fitting a binary logistic regression model using favorable versus unfavorable outcome as the response variable. For proportional odds modeling, the parameters were estimated by fitting ordinal logistic regression with the ordinal outcome (GOS or mRS) as the response variable. The entire process was repeated 1000 times, and the performance of each statistical approach was assessed by the proportion of 1000 analyses yielding significant results at the 5\% level. A 2-tailed $\mathrm{p}$ value $<0.05$ indicated statistical significance. Analyses were conducted in SAS version 9.2 (SAS Institute, Inc.) using standard procedures, as well as the logistic procedure for proportional odds modeling.

\section{Results}

The distribution of outcomes on the ordinal GOS and $\mathrm{mRS}$ and by tertiles of prognostic scores, according to treatment groups, was tabulated (Tables $2-5$ ). The number of favorable outcomes used to derive the absolute difference and odds ratios for the comparative treatment groups are summed over the 3 prognostic bands (Tables 4 and 5; breakpoint for the sliding method is indicated by values in boldface, and breakpoint for the median ap- 
TABLE 2: Original distribution of outcomes on the mRS and GOS for tirilazad and CONSCIOUS-1 trial data by treatment, age, or WFNS grade*

\begin{tabular}{|c|c|c|c|c|c|c|}
\hline Category & Death & Vegetative State & Severe Disability & Moderate Disability & Good Recovery & Total \\
\hline \multicolumn{7}{|l|}{ Tirilazad } \\
\hline \multicolumn{7}{|l|}{ treatment } \\
\hline vehicle & $236(17)$ & $22(2)$ & $140(10)$ & $196(14)$ & $764(56)$ & 1358 \\
\hline tirilazad & $342(16)$ & $37(2)$ & $284(13)$ & $266(12)$ & $1211(57)$ & 2140 \\
\hline total & 578 & 59 & 424 & 462 & 1975 & 3498 \\
\hline \multicolumn{7}{|l|}{ age in yrs } \\
\hline$\leq 51$ & $221(13)$ & $15(1)$ & $150(9)$ & $225(13)$ & $1143(65)$ & 1754 \\
\hline$>51$ & $357(20)$ & $44(3)$ & $274(16)$ & 237 (14) & $832(48)$ & 1744 \\
\hline \multicolumn{7}{|l|}{ WFNS grade $†$} \\
\hline good & $287(11)$ & $30(1)$ & $259(10)$ & $334(12)$ & $1799(66)$ & 2709 \\
\hline poor & $291(37)$ & $29(4)$ & $165(21)$ & $128(16)$ & $176(22)$ & 789 \\
\hline \multicolumn{7}{|l|}{ CONSCIOUS-1 } \\
\hline \multicolumn{7}{|l|}{ treatment } \\
\hline placebo & $4(4)$ & $2(2)$ & $24(25)$ & $45(47)$ & $21(22)$ & 96 \\
\hline clazosentan & $21(7)$ & $2(1)$ & $72(23)$ & $164(52)$ & $58(18)$ & 317 \\
\hline total & 25 & 4 & 96 & 209 & 79 & 413 \\
\hline \multicolumn{7}{|l|}{ age in yrs } \\
\hline$\leq 51$ & $10(5)$ & $1(1)$ & $44(21)$ & $123(59)$ & $32(15)$ & 210 \\
\hline$>51$ & $15(7)$ & $3(2)$ & $52(26)$ & $86(42)$ & $47(23)$ & 203 \\
\hline \multicolumn{7}{|l|}{ WFNS grade $†$} \\
\hline good & $13(4)$ & $1(0.32)$ & $56(18)$ & $171(56)$ & $72(23)$ & 313 \\
\hline poor & $12(12)$ & $3(3)$ & $40(40)$ & $38(38)$ & $7(7)$ & 100 \\
\hline
\end{tabular}

proach by values in cells shaded light gray). From these distributions, the number of favorable outcomes for the compared treatment groups and the absolute treatment or group differences with corresponding odds ratios for fixed and sliding dichotomy were calculated using the breakpoints defined above by 2 methods (sliding dichotomy and median split; Figs. 1-4).

An examination of Figs. 1-4 shows no consistent or sizable benefit in using the sliding dichotomy. Compared with fixed dichotomization, the sliding dichotomy decreased the treatment effect estimate from a $1 \%$ absolute increase in favorable outcomes to $0 \%$ for the tirilazad data, increased the treatment effect from $1 \%$ to $4 \%$ for the CONSCIOUS-1 data in favor of placebo, and decreased the treatment effect from $8 \%$ to $6 \%$ for the ISAT data. Similarly, when considering patient age, the sliding dichotomy decreased the difference between groups in the tirilazad trials and reversed the difference in the CONSCIOUS-1 data. For WFNS grade, the sliding dichotomy decreased the difference between groups in the tirilazad and CONSCIOUS-1 data. An analysis of randomly selected patients from the tirilazad or CONSCIOUS-1 data did not affect the results. For the CONSCIOUS-1 data, the median split method and conventional fixed dichotomy gave virtually identical results, whereas there were minor differences between the methods using the tirilazad and ISAT data.

For proportional odds modeling (shift analysis) using the 5-point GOS for tirilazad and CONSCIOUS-1 trial data and the 7-point mRS for the ISAT data, unadjusted models produced a good fit $\left(\chi^{2}=3.33,5 \mathrm{df}, \mathrm{p}=0.649\right)$; adjusted models produced a poor fit $\left(\chi^{2}=125.7,35 \mathrm{df}, \mathrm{p}<\right.$

TABLE 3: Original distribution of outcomes on the mRS and GOS for the ISAT data by treatment, age, or WFNS grade*

\begin{tabular}{lcccccccc}
\hline Treatment & Death & $\begin{array}{c}\text { Severe } \\
\text { Disability }\end{array}$ & $\begin{array}{c}\text { Moderately } \\
\text { Severe Disability }\end{array}$ & $\begin{array}{c}\text { Moderate } \\
\text { Disability }\end{array}$ & $\begin{array}{c}\text { Slight } \\
\text { Disability }\end{array}$ & $\begin{array}{c}\text { No Significant } \\
\text { Disability }\end{array}$ & $\begin{array}{c}\text { Good } \\
\text { Recovery }\end{array}$ & Total \\
\hline coiling & $80(8)$ & $27(3)$ & $28(3)$ & $104(10)$ & $\mathbf{2 5 1 ( 2 4 )}$ & $\mathbf{2 9 9 ( 2 8 )}$ & $\mathbf{2 5 9}(\mathbf{2 5})$ & 1048 \\
clipping & $101(10)$ & $37(4)$ & $42(4)$ & $138(13)$ & $\mathbf{2 4 8 ( 2 4 )}$ & $\mathbf{2 8 9 ( 2 8 )}$ & $\mathbf{1 8 6 ( 1 8 )}$ & 1041 \\
total & 181 & 64 & 70 & 242 & $\mathbf{4 9 9}$ & $\mathbf{5 8 8}$ & $\mathbf{4 4 5}$ & 2089 \\
\hline
\end{tabular}

* Columns in boldface correspond to the definition of good outcome when using the dichotomous GOS or mRS. 
TABLE 4: Distribution of outcomes on the mRS and GOS for the tirilazad and CONSCIOUS-1 data in 3 prognostic tiers based on treatment, age, or WFNS grade*

\begin{tabular}{|c|c|c|c|c|c|c|}
\hline Category & Death & Vegetative State & Severe Disability & Moderate Disability & Good Recovery & Total \\
\hline \multicolumn{7}{|l|}{ Tirilazad } \\
\hline \multicolumn{7}{|l|}{ treatment } \\
\hline \multicolumn{7}{|l|}{ worst } \\
\hline vehicle & $153(33)$ & $11(2)$ & $81(18)$ & $78(17)$ & $137(30)$ & 460 \\
\hline tirilazad & $202(29)$ & $30(4)$ & $143(20)$ & $103(15)$ & $220(32)$ & 698 \\
\hline total & 355 & 41 & 224 & 181 & 357 & 1158 \\
\hline \multicolumn{7}{|l|}{ intermediate } \\
\hline vehicle & $46(11)$ & $8(2)$ & $40(9)$ & $64(15)$ & $268(63)$ & 426 \\
\hline tirilazad & $83(13)$ & $3(0.5)$ & $72(11)$ & $90(14)$ & $403(62)$ & 651 \\
\hline total & 129 & 11 & 112 & 154 & 671 & 1077 \\
\hline \multicolumn{7}{|l|}{ best } \\
\hline vehicle & $32(7)$ & $2(0.45)$ & $15(3)$ & $47(11)$ & $346(78)$ & 442 \\
\hline tirilazad & $46(6)$ & $4(0.5)$ & $52(7)$ & $67(9)$ & $566(77)$ & 735 \\
\hline total & 78 & 6 & 67 & 114 & 912 & 1177 \\
\hline \multicolumn{7}{|l|}{ age in yrs } \\
\hline \multicolumn{7}{|l|}{ worst } \\
\hline$\leq 51$ & $137(26)$ & $8(2)$ & $77(14)$ & $103(19)$ & $209(39)$ & 534 \\
\hline$>51$ & $241(30)$ & $34(4)$ & $162(20)$ & $105(13)$ & $272(33)$ & 814 \\
\hline total & 378 & 42 & 239 & 208 & 481 & 1348 \\
\hline \multicolumn{7}{|l|}{ intermediate } \\
\hline$\leq 51$ & $52(9)$ & $3(0.54)$ & $46(8)$ & 59 (11) & $396(71)$ & 556 \\
\hline$>51$ & $65(13)$ & $8(2)$ & 54 (11) & $76(15)$ & $291(59)$ & 494 \\
\hline total & 117 & 11 & 100 & 135 & 687 & 1050 \\
\hline \multicolumn{7}{|l|}{ best } \\
\hline$\leq 51$ & $26(4)$ & $3(0.5)$ & $21(3)$ & $55(9)$ & $516(83)$ & 621 \\
\hline$>51$ & $41(10)$ & $2(0.5)$ & $43(11)$ & $51(13)$ & $256(65)$ & 393 \\
\hline total & 67 & 5 & 64 & 106 & 772 & 1014 \\
\hline \multicolumn{7}{|l|}{$\begin{array}{c}\text { WFNS grade } \\
\text { worst }\end{array}$} \\
\hline good & $140(16)$ & $21(2)$ & 119 (13) & $132(15)$ & $475(54)$ & 887 \\
\hline poor & $147(43)$ & $14(4)$ & 77 (22) & $36(10)$ & $70(20)$ & 344 \\
\hline total & 287 & 35 & 196 & 168 & 545 & 1231 \\
\hline \multicolumn{7}{|l|}{ intermediate } \\
\hline good & $93(10)$ & $5(0.5)$ & $79(9)$ & $113(13)$ & $599(67)$ & 889 \\
\hline poor & $100(35)$ & $10(3)$ & $55(19)$ & $56(20)$ & $66(23)$ & 287 \\
\hline total & 193 & 15 & 134 & 169 & 665 & 1176 \\
\hline \multicolumn{7}{|l|}{ best } \\
\hline good & $49(6)$ & $4(0.4)$ & $46(5)$ & $79(9)$ & $693(80)$ & 871 \\
\hline poor & $33(25)$ & $4(3)$ & $27(20)$ & $33(25)$ & 37 (28) & 134 \\
\hline total & 82 & 8 & 73 & 112 & 730 & 1005 \\
\hline \multicolumn{7}{|l|}{ CONSCIOUS-1 } \\
\hline \multicolumn{7}{|l|}{ treatment } \\
\hline \multicolumn{7}{|l|}{ worst } \\
\hline placebo & $4(11)$ & $2(6)$ & $12(34)$ & $15(43)$ & $2(6)$ & 35 \\
\hline clazosentan & $11(11)$ & $2(2)$ & 37 (37) & $39(39)$ & $12(12)$ & 101 \\
\hline total & 15 & 4 & 49 & 54 & 14 & 136 \\
\hline
\end{tabular}


TABLE 4: Distribution of outcomes on the mRS and GOS for the tirilazad and CONSCIOUS-1 data in 3 prognostic tiers based on treatment, age, or WFNS grade* (continued)

\begin{tabular}{|c|c|c|c|c|c|c|}
\hline Category & Death & Vegetative State & Severe Disability & Moderate Disability & Good Recovery & Total \\
\hline \multicolumn{7}{|c|}{ CONSCIOUS-1 (continued) } \\
\hline \multicolumn{7}{|c|}{ treatment (continued) } \\
\hline \multicolumn{7}{|c|}{ intermediate } \\
\hline placebo & 0 & 0 & $8(23)$ & $19(56)$ & $7(21)$ & 34 \\
\hline clazosentan & $10(7)$ & 0 & $26(19)$ & $75(54)$ & $27(20)$ & 138 \\
\hline total & 10 & 0 & 34 & 94 & 34 & 172 \\
\hline \multicolumn{7}{|l|}{ best } \\
\hline placebo & 0 & 0 & $4(15)$ & $11(41)$ & $12(44)$ & 27 \\
\hline clazosentan & 0 & 0 & $8(11)$ & $49(65)$ & $18(24)$ & 75 \\
\hline total & 0 & 0 & 12 & 60 & 30 & 102 \\
\hline \multicolumn{7}{|l|}{ age in yrs } \\
\hline \multicolumn{7}{|l|}{ worst } \\
\hline$\leq 51$ & $6(11)$ & $1(2)$ & $21(37)$ & $27(47)$ & $2(4)$ & 57 \\
\hline$>51$ & $9(11)$ & $3(4)$ & $28(35)$ & $27(34)$ & $12(15)$ & 79 \\
\hline total & 15 & 4 & 49 & 54 & 14 & 136 \\
\hline \multicolumn{7}{|l|}{ intermediate } \\
\hline$\leq 51$ & $4(5)$ & 0 & $16(19)$ & $53(62)$ & $12(14)$ & 85 \\
\hline$>51$ & $6(7)$ & 0 & $18(21)$ & $41(47)$ & $22(25)$ & 87 \\
\hline total & 10 & 0 & 34 & 94 & 34 & 172 \\
\hline \multicolumn{7}{|l|}{ best } \\
\hline$\leq 51$ & 0 & 0 & $7(10)$ & $43(63)$ & $18(26)$ & 68 \\
\hline$>51$ & 0 & 0 & $5(15)$ & $17(50)$ & $12(35)$ & 34 \\
\hline total & 0 & 0 & 12 & 60 & 30 & 102 \\
\hline \multicolumn{7}{|l|}{ WFNS grade } \\
\hline \multicolumn{7}{|l|}{ worst } \\
\hline good & $6(7)$ & $1(1)$ & $20(25)$ & $37(46)$ & $17(21)$ & 81 \\
\hline poor & $5(11)$ & $3(7)$ & $20(45)$ & $15(34)$ & $1(2)$ & 44 \\
\hline total & 11 & 4 & 40 & 52 & 18 & 125 \\
\hline \multicolumn{7}{|l|}{ intermediate } \\
\hline good & $7(5)$ & 0 & $23(18)$ & $74(58)$ & $24(19)$ & 128 \\
\hline poor & $5(15)$ & 0 & $10(29)$ & $16(47)$ & $3(9)$ & 34 \\
\hline total & 12 & 0 & 33 & 90 & 27 & 162 \\
\hline \multicolumn{7}{|l|}{ best } \\
\hline good & 0 & 0 & $12(12)$ & $60(59)$ & $30(29)$ & 102 \\
\hline poor & $2(10)$ & 0 & $10(48)$ & $6(29)$ & $3(14)$ & 21 \\
\hline total & 2 & 0 & 22 & 66 & 33 & 123 \\
\hline
\end{tabular}

* Values in boldface correspond to the definition of a good outcome when using the conventional sliding definition of outcome, and cells with light gray shading correspond to the cut points used for the median split method.

0.0001) for the ISAT data because the proportional odds assumption was not valid ( $\mathrm{p}<0.0001$; Table 6). Using tirilazad data, the unadjusted and adjusted models resulted in poor fits $\left(\chi^{2}=9.92,3 \mathrm{df}, \mathrm{p}=0.019\right.$; and $\chi^{2}=41.8$, $15 \mathrm{df}, \mathrm{p}=0.0002)$ due to an invalid proportional odds assumption. Using CONSCIOUS-1 data, the unadjusted model produced a good fit $\left(\chi^{2}=2.62,3 \mathrm{df}, \mathrm{p}=0.454\right)$ but the adjusted model produced a poor fit $\left(\chi^{2}=292.4,15\right.$ df, $\mathrm{p}<0.0001)$ again because the proportional odds assumption was not valid $(\mathrm{p}=0.528)$. Collapsing death and vegetative state into a single category to make a 4-point ordinal outcome scale in the CONSCIOUS-1 data led to improved fit with an adjusted proportional odds model $\left(\chi^{2}\right.$ $=7.11,10 \mathrm{df}, \mathrm{p}=0.72)$. The point estimate of the common odds ratio for the clazosentan treatment effect was 0.87 (95\% CI 0.56-1.35, $\mathrm{p}=0.535)$.

A comparison of fixed dichotomy, sliding dichotomy, and shift analysis (proportional odds modeling), whether adjusted for covariates or not, did not reveal that any particular method was substantially different from the others (Table 6). In the CONSCIOUS-1 data, the sliding dichotomy was more sensitive than the proportional odds 


\section{Sliding dichotomy for SAH}

TABLE 5: Distribution of outcomes on the mRS and GOS for the ISAT data in 3 prognostic tiers based on treatment, age, or WFNS grade*

\begin{tabular}{|c|c|c|c|c|c|c|c|c|}
\hline Category & Death & $\begin{array}{c}\text { Severe } \\
\text { Disability }\end{array}$ & $\begin{array}{c}\text { Moderately } \\
\text { Severe Disability }\end{array}$ & $\begin{array}{l}\text { Moderate } \\
\text { Disability }\end{array}$ & $\begin{array}{c}\text { Slight } \\
\text { Disability }\end{array}$ & $\begin{array}{c}\text { No Significant } \\
\text { Disability }\end{array}$ & $\begin{array}{c}\text { Good } \\
\text { Recovery }\end{array}$ & Total \\
\hline \multicolumn{9}{|l|}{ worst } \\
\hline coiling & $45(14)$ & $11(3)$ & $13(4)$ & $40(12)$ & $75(23)$ & $87(27)$ & $56(17)$ & 327 \\
\hline clipping & $49(15)$ & $23(7)$ & $14(4)$ & $50(15)$ & $63(19)$ & $74(23)$ & $51(16)$ & 324 \\
\hline total & 94 & 34 & 27 & 90 & 138 & 161 & 107 & 651 \\
\hline \multicolumn{9}{|l|}{ intermediate } \\
\hline coiling & $22(6)$ & $12(4)$ & $10(3)$ & 37 (11) & $85(25)$ & $92(27)$ & $80(24)$ & 338 \\
\hline clipping & $38(11)$ & $8(2)$ & $13(4)$ & $43(12)$ & $82(24)$ & $105(30)$ & $59(17)$ & 348 \\
\hline total & 60 & 20 & 23 & 80 & 167 & 197 & 139 & 686 \\
\hline \multicolumn{9}{|l|}{ best } \\
\hline coiling & $13(3)$ & $4(1)$ & $5(1)$ & $27(7)$ & $91(24)$ & $120(31)$ & $122(32)$ & 382 \\
\hline clipping & $14(4)$ & $6(2)$ & $15(4)$ & $45(12)$ & $103(28)$ & $110(30)$ & $76(20)$ & 369 \\
\hline total & 27 & 10 & 20 & 72 & 194 & 230 & 198 & 751 \\
\hline
\end{tabular}

model and the fixed dichotomy ( $p=0.25$ vs $p=0.53$ vs $\mathrm{p}=0.98$, respectively). The calculated effect sizes for the 3 methods were quite close and similar in magnitude and sometimes in opposite directions.

In Table 7 we compare results of the actual trial and simulated tirilazad and ISAT data. For tirilazad, both sliding dichotomy (OR changed from 0.97 to 1.13) and proportional odds modeling (OR changed from 0.97 to 1.12) increased the effect size of treatment. Results from the 1000 analyses showed similar nonsignificant increases. For the ISAT, sliding dichotomy and fixed dichotomy produced about the same effect, but the proportional odds modeling improved the effect of mode of treatment (clipping compared with coiling, OR changed from 0.67 to 0.70) as compared with fixed dichotomization. Using the

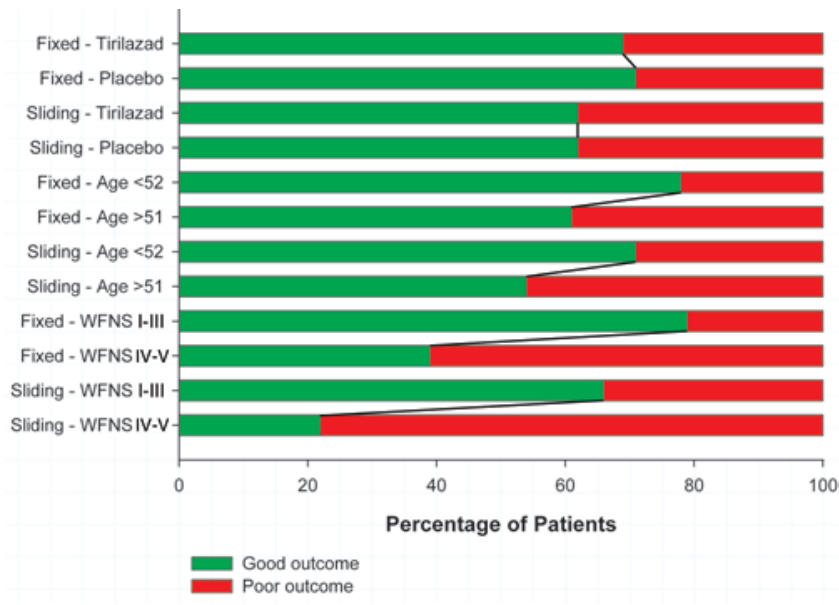

FIG. 1. Bar graph showing clinical outcome in the tirilazad studies according to the GOS and comparing the tirilazad and placebo groups or based on patient age or WFNS admission grade. Each set of bars demonstrates the difference between the groups when assessed using conventional fixed dichotomization of the GOS or utilizing the sliding dichotomy implemented with a median split method. simulated data, proportional odds modeling still outperformed fixed dichotomy, with an increased effect size of mode of treatment (OR changed from 0.66 to 0.71 ), whereas sliding dichotomy reduced the effect size (OR changed from 0.66 to 0.64 ). Moreover, a higher proportion of the 1000 analyses yielded significant results at the $5 \%$ level for these different methods.

\section{Discussion}

The most common method of analyzing outcome in randomized clinical trials in stroke, $\mathrm{SAH}$, and head injury has been to dichotomize outcome, usually per the GOS, into favorable and unfavorable categories. This simplifies the analysis but may reduce statistical power.

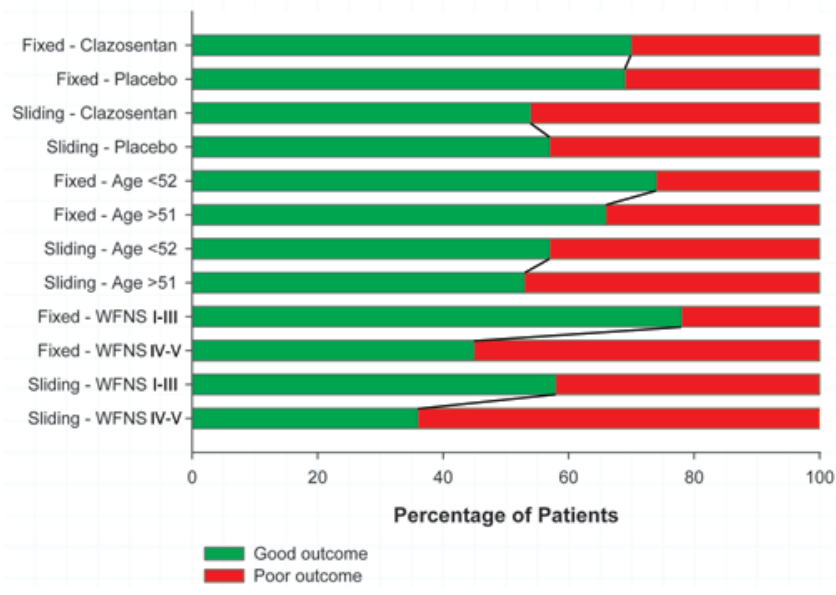

FIG. 2. Bar graph showing clinical outcome in the CONSCIOUS-1 trial according to the GOS and comparing the clazosentan and placebo groups or based on patient age or WFNS admission grade. Each set of bars shows the difference between the groups when assessed using conventional fixed dichotomization of the GOS or utilizing the sliding dichotomy implemented with a median split method. 


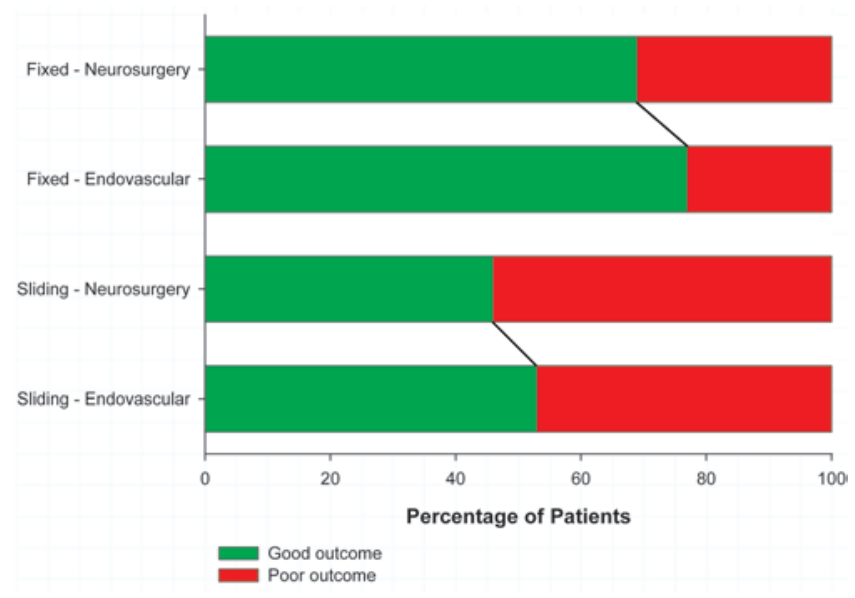

FIG. 3. Bar graph demonstrating clinical outcome in the ISAT according to the $\mathrm{mRS}$ and comparing the neurosurgical clipping or endovascular coiling groups. Each set of bars shows the difference between the groups when assessed using conventional fixed dichotomization of the GOS or mRS or utilizing the sliding dichotomy implemented with a median split method.

Other methods, such as sliding dichotomy (prognosis-adjusted end point or responder analysis), global statistics, and proportional odds modeling, have been put forward as alternatives to fixed dichotomization. ${ }^{2,11,14,17}$

Sliding dichotomy has increased statistical power and reduced sample sizes in a post hoc analysis of some head injury trials. ${ }^{17}$ In the clinical trials reanalyzed in the present study, sliding dichotomy did not increase the difference in outcomes among patients entered into the ISAT, perhaps because the main shift in outcome was already accounted for by a shift across the moderate disability to slight disability line, whereas shifts between other categories must
TABLE 6: Odds ratios for fixed and sliding dichotomy and shift analysis on each data set*

\begin{tabular}{|c|c|c|c|}
\hline Study \& Method & Covariates & OR (95\% Cl) & p Value \\
\hline \multicolumn{4}{|l|}{ Tirilazad } \\
\hline \multirow[t]{2}{*}{ fixed dichotomy } & unadjusted & $0.92(0.80-1.07)$ & 0.294 \\
\hline & adjusted & $0.89(0.76-1.05)$ & 0.178 \\
\hline \multirow[t]{2}{*}{ sliding dichotomy } & unadjusted & $1.02(0.87-1.19)$ & 0.791 \\
\hline & adjusted & $1.00(0.86-1.18)$ & 0.971 \\
\hline \multirow[t]{2}{*}{ shift analysis } & unadjusted & $1.01(0.89-1.15)$ & 0.895 \\
\hline & adjusted & $0.98(0.86-1.13)$ & 0.809 \\
\hline \multicolumn{4}{|l|}{ CONSCIOUS-1 } \\
\hline \multirow[t]{2}{*}{ fixed dichotomy } & unadjusted & $1.06(0.65-1.74)$ & 0.806 \\
\hline & adjusted & $1.01(0.59-1.73)$ & 0.978 \\
\hline \multirow[t]{2}{*}{ sliding dichotomy } & unadjusted & $0.85(0.52-1.39)$ & 0.518 \\
\hline & adjusted & $0.73(0.42-1.26)$ & 0.254 \\
\hline \multirow[t]{2}{*}{ shift analysis } & unadjusted & $0.93(0.61-1.43)$ & 0.749 \\
\hline & adjusted & $0.87(0.56-1.35)$ & 0.528 \\
\hline \multicolumn{4}{|l|}{ ISAT } \\
\hline \multirow[t]{2}{*}{ fixed dichotomy } & unadjusted & $0.67(0.55-0.82)$ & $<0.0001$ \\
\hline & adjusted & $0.66(0.54-0.81)$ & $<0.0001$ \\
\hline \multirow[t]{2}{*}{ sliding dichotomy } & unadjusted & $0.67(0.53-0.85)$ & 0.0003 \\
\hline & adjusted & $0.66(0.52-0.84)$ & 0.0002 \\
\hline \multirow[t]{2}{*}{ shift analysis } & unadjusted & $0.70(0.60-0.82)$ & $<0.0001$ \\
\hline & adjusted & $0.69(0.60-0.81)$ & $<0.0001$ \\
\hline
\end{tabular}

* Covariates adjusted for were age, sex, WFNS grade, and SAH clot thickness.

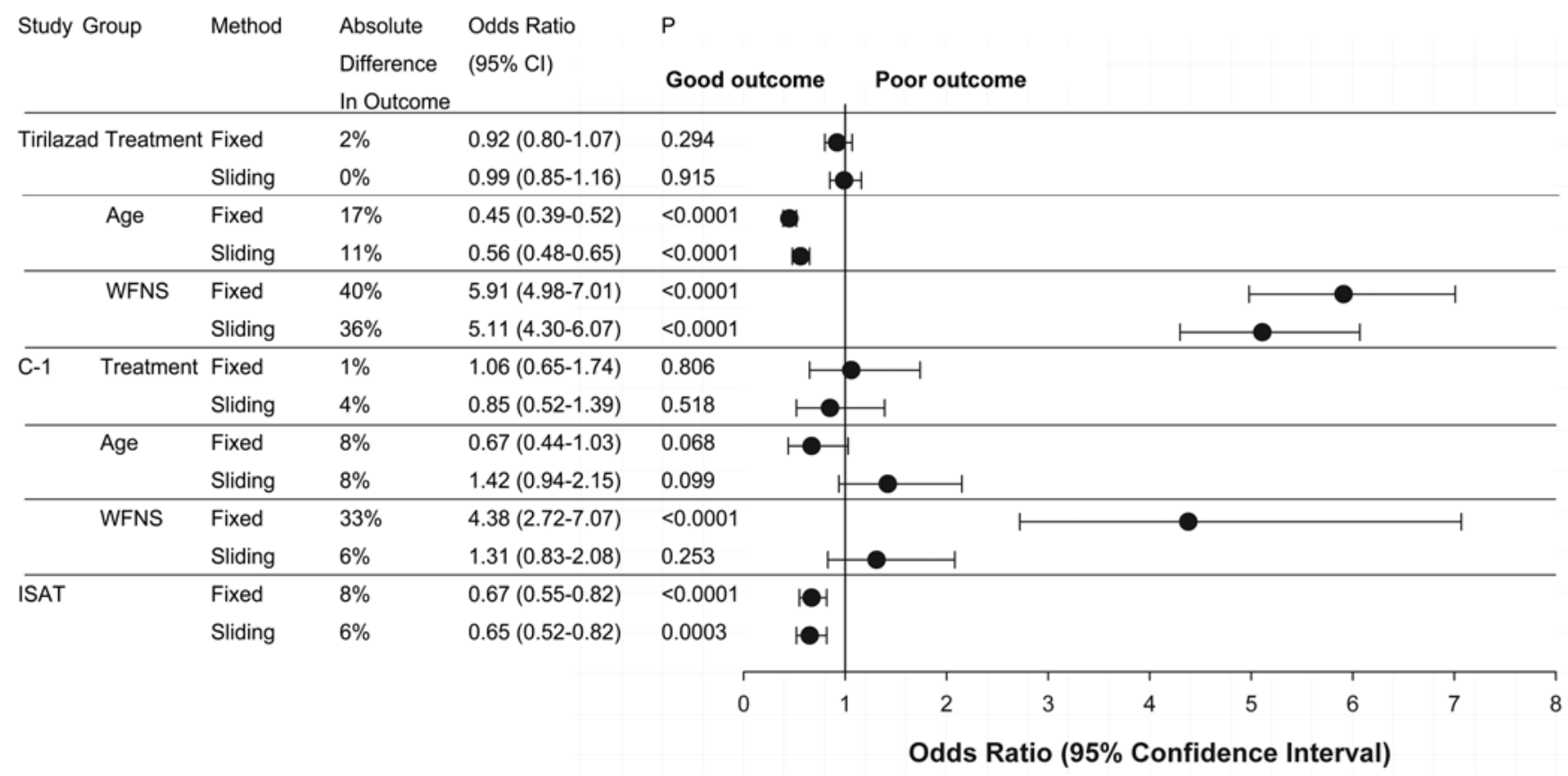

Fig. 4. Estimation of effect size using fixed and sliding dichotomy implemented with a median split method for each data set. Differences between outcomes by the 2 methods are of variable size and inconsistent. C-1 = CONSCIOUS-1. 
TABLE 7: Comparison of treatment effects on the GOS and mRS, according to conventional dichotomy, sliding dichotomy, and proportional odds logistic regression using trial or simulated data*

\begin{tabular}{|c|c|c|c|c|c|c|}
\hline Study & $\begin{array}{l}\text { Unadjusted OR } \\
\qquad(95 \% \mathrm{Cl})\end{array}$ & Wald Statistic & p Value & $\begin{array}{l}\text { Adjusted OR } \\
(95 \% \mathrm{Cl})\end{array}$ & Wald Statistic & $\mathrm{p}$ Value \\
\hline \multicolumn{7}{|l|}{ Tirilazad } \\
\hline \multicolumn{7}{|l|}{ trial data } \\
\hline fixed dichotomy & $0.92(0.80-1.07)$ & 1.101 & 0.294 & $0.89(0.76-1.05)$ & 1.813 & 0.178 \\
\hline sliding dichotomy & $1.02(0.87-1.19)$ & 0.070 & 0.791 & $1.00(0.86-1.18)$ & 0.002 & 0.971 \\
\hline proportional odds & $1.01(0.89-1.15)$ & 0.017 & 0.895 & $0.98(0.86-1.13)$ & 0.051 & 0.809 \\
\hline \multicolumn{7}{|l|}{ simulated data } \\
\hline fixed dichotomy & $0.97(0.73-1.28)$ & 0.064 & 0.800 & $0.98(0.71-1.35)$ & 0.022 & 0.882 \\
\hline sliding dichotomy & $1.13(0.85-1.52)$ & 0.714 & 0.398 & $1.12(0.83-1.51)$ & 0.535 & 0.465 \\
\hline proportional odds & $1.12(0.88-1.43)$ & 0.811 & 0.368 & $1.18(0.90-1.53)$ & 1.437 & 0.231 \\
\hline \multicolumn{7}{|l|}{ ISAT } \\
\hline \multicolumn{7}{|l|}{ trial data } \\
\hline fixed dichotomy & $0.67(0.55-0.82)$ & 15.76 & $<0.001$ & $0.66(0.54-0.81)$ & 16.34 & $<0.001$ \\
\hline sliding dichotomy & $0.67(0.53-0.85)$ & 11.55 & 0.001 & $0.66(0.53-0.84)$ & 12.00 & 0.001 \\
\hline proportional odds & $0.70(0.60-0.82)$ & 20.81 & $<0.001$ & $0.69(0.60-0.81)$ & 21.71 & $<0.001$ \\
\hline \multicolumn{7}{|l|}{ simulated data } \\
\hline fixed dichotomy & $0.66(0.50-0.87)$ & 8.559 & 0.003 & $0.64(0.48-0.86)$ & 9.134 & 0.003 \\
\hline sliding dichotomy & $0.64(0.46-0.89)$ & 6.902 & 0.009 & $0.63(0.45-0.89)$ & 6.952 & 0.008 \\
\hline proportional odds & $0.71(0.57-0.88)$ & 9.095 & 0.003 & $0.72(0.58-0.89)$ & 8.515 & 0.004 \\
\hline
\end{tabular}

* An OR > 1 indicates an adverse treatment effect. Covariates adjusted for in the tirilazad trials were age, WFNS grade, SAH clot thickness, and hypertension. Covariates adjusted for in the ISAT were age, WFNS grade, and sex.

have been less after accounting for prognostic factors. In the tirilazad and CONSCIOUS-1 data, there was no significant benefit of drug treatment on the dichotomous GOS, which raises the additional possibility that these drugs (tirilazad and clazosentan) have no effect, and no method of analysis will demonstrate a benefit.

We thought that if we dichotomized the tirilazad and CONSCIOUS-1 data based on a known prognostic factor and then constructed a prognosis-adjusted end point based on other prognostic factors, then increased statistical power would result from a sliding dichotomy. Again, when considering patient age or WFNS grade, no consistent marked benefits were observed with a sliding dichotomy. The distribution of patients and the changes that occur between categories must therefore be very similar among the age or WFNS strata.

The third method assessed here was proportional odds modeling. This method depends on the proportional odds assumption but may still provide a robust estimate of treatment effect even when this assumption is not met. Proportional odds modeling has been used in both head injury and stroke trials. ${ }^{2,11}$ The proportional odds model explores ordering of the outcome scale, but the sliding dichotomy focuses on that part of the ordered scale that is of greatest clinical relevance to a particular patient. The proportional odds model sensitively detects a shift over the entire ordered scale. Note that the sliding dichotomy mirrors clinical thinking, more so than does the proportional odds modeling technique. By this we mean that the treating physician might consider the only favorable outcome to be good recovery for a young patient with WFNS Grade I.
Traditional dichotomization of the GOS would also include moderate disability, which could be considered worse than the outcome that should be achieved. Similarly, we might consider the successful treatment of an elderly patient with WFNS Grade IV or V to be the upper tier of severe disability. This outcome would still be considered unfavorable by traditional dichotomization. Sliding dichotomy can account for these differences. In the trials included here, the proportional odds assumption was violated in adjusted models; therefore, proportional odds modeling might not be a preferred method over sliding or fixed dichotomy based on strict statistical criteria even though it appeared to produce a more powerful result.

\section{Conclusions}

Data from 7 clinical trials of patients with SAH showed that, in general, fixed dichotomy and proportional odds modeling performed better than sliding dichotomy, although the improvements were modest and not often statistically significant. That said, a theoretical basis for both shift analysis and sliding dichotomy (the latter uses prognostic information) appears preferable to fixed dichotomy to capture more information about the response to an intervention. Recent studies based on simulations, ${ }^{13}$ the reanalysis of a major head injury study, ${ }^{18}$ and the prospective analysis of a major stroke trial ${ }^{19}$ have all demonstrated very substantial efficiency gains from the application of ordinal analysis. Perhaps the GOS and mRS, which were not developed for use in SAH, do not assess outcomes that are relevant to patients with SAH. It is im- 
portant that future research explores the conditions under which such efficiency gains can be achieved in practice.

\section{Disclosure}

Actelion Pharmaceuticals, Ltd., was the sponsor of the CONSCIOUS-1 trial and they provided financial support for the exploratory analysis described in this article. The data analysis and writing are the work of the authors. Dr. Macdonald is the chief scientific officer for and has direct stock ownership in Edge Therapeutics, Inc.; is a consultant for Actelion Pharmaceuticals, Ltd.; and receives grant funding from Physicians Services Incorporated Foundation and the Heart and Stroke Foundation of Canada. Dr. Kassell has ownership in InSightec. Dr. Molyneux is a consultant for Actelion Pharmaceuticals, Ltd., and Micrus Endovascular Corp. and receives grant support from the Medical Research Council UK and Cerecyte Coil Trial (Micrus Endovascular).

Author contributions to the study and manuscript preparation include the following. Conception and design: Macdonald, Murray. Acquisition of data: Macdonald, Kerr, Molyneux. Analysis and interpretation of data: Macdonald, Ilodigwe, Murray, Kassell, Torner, Molyneux. Drafting the article: Macdonald, Ilodigwe, Murray. Critically revising the article: all authors. Reviewed submitted version of manuscript: all authors. Approved the final version of the manuscript on behalf of all authors: Macdonald. Statistical analysis: Macdonald, Ilodigwe, Murray, Torner. Administrative/technical/ material support: Macdonald. Study supervision: Macdonald.

\section{References}

1. Bath PM, Gray LJ, Collier T, Pocock S, Carpenter J: Can we improve the statistical analysis of stroke trials? Statistical reanalysis of functional outcomes in stroke trials. Stroke 38: 1911-1915, 2007

2. Bolland K, Sooriyarachchi MR, Whitehead J: Sample size review in a head injury trial with ordered categorical responses. Stat Med 17:2835-2847, 1998

3. Bonita R, Beaglehole R: Recovery of motor function after stroke. Stroke 19:1497-1500, 1988

4. Hacke W, Kaste M, Fieschi C, von Kummer R, Davalos A, Meier D, et al: Randomised double-blind placebo-controlled trial of thrombolytic therapy with intravenous alteplase in acute ischaemic stroke (ECASS II). Lancet 352:1245-1251, 1998

5. Haley EC Jr, Kassell NF, Alves WM, Weir BK, Hansen CA: Phase II trial of tirilazad in aneurysmal subarachnoid hemorrhage. A report of the Cooperative Aneurysm Study. J Neurosurg 82:786-790, 1995

6. Haley EC Jr, Kassell NF, Apperson-Hansen C, Maile MH, Alves WM: A randomized, double-blind, vehicle-controlled trial of tirilazad mesylate in patients with aneurysmal subarachnoid hemorrhage: a cooperative study in North America. J Neurosurg 86:467-474, 1997

7. Jennett B, Bond M: Assessment of outcome after severe brain damage. Lancet 1:480-484, 1975

8. Kassell NF, Haley ECJ Jr, Apperson-Hansen C, Alves WM: Randomized, double-blind, vehicle-controlled trial of tirilazad mesylate in patients with aneurysmal subarachnoid hemorrhage: a cooperative study in Europe, Australia, and New Zealand. J Neurosurg 84:221-228, 1996

9. Lanzino G, Kassell NF: Double-blind, randomized, vehiclecontrolled study of high-dose tirilazad mesylate in women with aneurysmal subarachnoid hemorrhage. Part II. A cooperative study in North America. J Neurosurg 90:1018-1024, 1999

10. Lanzino G, Kassell NF, Dorsch NW, Pasqualin A, Brandt L, Schmiedek P, et al: Double-blind, randomized, vehicle-controlled study of high-dose tirilazad mesylate in women with aneurysmal subarachnoid hemorrhage. Part I. A cooperative study in Europe, Australia, New Zealand, and South Africa. J Neurosurg 90:1011-1017, 1999
11. Lees KR, Asplund K, Carolei A, Davis SM, Diener HC, Kaste $\mathrm{M}$, et al: Glycine antagonist (gavestinel) in neuroprotection (GAIN International) in patients with acute stroke: a randomised controlled trial. Lancet 355:1949-1954, 2000

12. Macdonald RL, Kassell NF, Mayer S, Ruefenacht D, Schmiedek $\mathrm{P}$, Weidauer S, et al: Clazosentan to overcome neurological ischemia and infarction occurring after subarachnoid hemorrhage (CONSCIOUS-1): randomized, double-blind, placebocontrolled phase 2 dose-finding trial. Stroke 39:3015-3021, 2008

13. McHugh GS, Butcher I, Steyerberg EW, Marmarou A, Lu J, Lingsma HF, et al: A simulation study evaluating approaches to the analysis of ordinal outcome data in randomized controlled trials in traumatic brain injury: results from the IMPACT Project. Clin Trials 7:44-57, 2010

14. Mendelow AD, Gregson BA, Fernandes HM, Murray GD, Teasdale GM, Hope DT, et al: Early surgery versus initial conservative treatment in patients with spontaneous supratentorial intracerebral haematomas in the International Surgical Trial in Intracerebral Haemorrhage (STICH): a randomised trial. Lancet 365:387-397, 2005

15. Molyneux A, Kerr R, Stratton I, Sandercock P, Clarke M, Shrimpton J, et al: International Subarachnoid Aneurysm Trial (ISAT) of neurosurgical clipping versus endovascular coiling in 2143 patients with ruptured intracranial aneurysms: a randomised trial. Lancet 360:1267-1274, 2002

16. Molyneux AJ, Kerr RS, Yu LM, Clarke M, Sneade M, Yarnold JA, et al: International subarachnoid aneurysm trial (ISAT) of neurosurgical clipping versus endovascular coiling in 2143 patients with ruptured intracranial aneurysms: a randomised comparison of effects on survival, dependency, seizures, rebleeding, subgroups, and aneurysm occlusion. Lancet 366:809-817, 2005

17. Murray GD, Barer D, Choi S, Fernandes H, Gregson B, Lees $\mathrm{KR}$, et al: Design and analysis of phase III trials with ordered outcome scales: the concept of the sliding dichotomy. J Neurotrauma 22:511-517, 2005

18. Roozenbeek B, Lingsma HF, Perel P, Edwards P, Roberts I, Murray GD, et al: The added value of ordinal analysis in clinical trials: an example in traumatic brain injury. Crit Care 15: R127, 2011

19. Sandset EC, Bath PM, Boysen G, Jatuzis D, Kõrv J, Lüders S, et al: The angiotensin-receptor blocker candesartan for treatment of acute stroke (SCAST): a randomised, placebo-controlled, double-blind trial. Lancet 377:741-750, 2011

20. Saver JL: Novel end point analytic techniques and interpreting shifts across the entire range of outcome scales in acute stroke trials. Stroke 38:3055-3062, 2007

21. Senn S, Julious S: Measurement in clinical trials: a neglected issue for statisticians? Stat Med 28:3189-3209, 2009

22. Wilson JT, Pettigrew LE, Teasdale GM: Structured interviews for the Glasgow Outcome Scale and the extended Glasgow Outcome Scale: guidelines for their use. J Neurotrauma 15: 573-585, 1998

Manuscript submitted August 10, 2011.

Accepted September 4, 2012.

Portions of this work were presented at the 44th Annual Congress of the Canadian Neurological Sciences Federation held on June 9-12, 2009, in Halifax, Nova Scotia, and were published as an abstract.

Please include this information when citing this paper: published online October 5, 2012; DOI: 10.3171/2012.9.JNS111383.

Address correspondence to: R. Loch Macdonald, M.D., Ph.D., Division of Neurosurgery, St. Michael's Hospital, University of Toronto, 30 Bond Street, Toronto, Ontario, Canada M5B 1W8. email: macdonaldlo@smh.ca. 